Fixed Point Theory, 22(2021), No. 1, 59-74

DOI: $10.24193 /$ fpt-ro.2021.1.04

http://www.math.ubbcluj.ro/ nodeacj/sfptcj.html

\title{
FIXED DISCS IN QUASI-METRIC SPACES
}

\author{
HASSEN AYDI*, NIHAL TAŞ**, NIHAL YILMAZ ÖZGÜR ${ }^{* * *}$ AND NABIL MLAIKI****
}

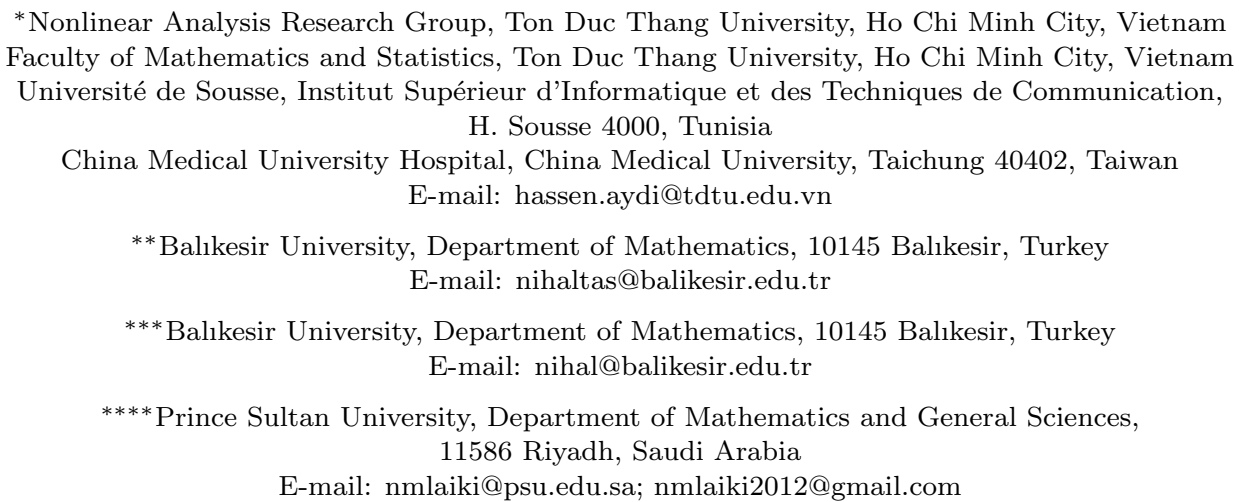

Abstract. In this paper, we present some results of fixed disc and common fixed disc in quasi-metric spaces, under some very interesting contractions. Obtained results are supported by illustrative examples.

Key Words and Phrases: Fixed disc, common fixed disc, quasi-metric space, contraction.

2020 Mathematics Subject Classification: 54H25, 47H09, 47H10.

Acknowledgement. The authors would like to thank the anonymous referee for her/his comments that helped us improve this article. The second and third authors were supported by Balýkesir University Research Grant no: 2018/021. The fourth author would like to thank Prince Sultan University for funding this work through research group Nonlinear Analysis Methods in Applied Mathematics (NAMAM) group number RG-DES-2017-01-17.

\section{REFERENCES}

[1] H. Afshari, S. Kalantari, H. Aydi, Fixed point results for generalized $\alpha-\psi$-Suzuki-contractions in quasi-b-metric-like spaces, Asian-Eur. J. Math., 11(2018), no. 1, 1850012 (12 pages).

[2] H.H. Alsulami, E. Karapınar, F. Khojasteh, A.F. Roldán-López-de-Hierro, A proposal to the study of contractions in quasi-metric spaces, Discrete Dyn. Nat. Soc., (2014), Article ID 269286, 10 pages.

[3] H. Aydi, $\alpha$-implicit contractive pair of mappings on quasi b-metric spaces and an application to integral equations, J. Nonlinear Convex Anal., 17(2016), no. 12, 2417-2433. 
[4] H. Aydi, A. Felhi, E. Karapınar, F.A. Alojail, Fixed points on quasi-metric spaces via simulation functions and consequences, J. Math. Anal., 9(2018), no. 2, 10-24.

[5] H. Aydi, M. Jellali, E. Karapinar, On fixed point results for $\alpha$-implicit contractions in quasimetric spaces and consequences, Nonlinear Anal., Model. Control, 21(2016), no. 1, 40-56.

[6] H. Aydi, N. Taş, N.Y. Özgür, N. Mlaiki, Fixed-discs in rectangular metric spaces, Symmetry, 11(2019), no. 2, 294.

[7] S. Banach, Sur les operations dans les ensembles abstraits et leur application aux equations integrals, Fundam. Math., 2(1922), 133-181.

[8] V. Berinde, Contracţii Generalizate şi Aplicaţii, 22, Editura Cub Press, Baia Mare, 1997.

[9] N. Bilgili, E. Karapinar, B. Samet, Generalized $\alpha-\psi$ contractive mappings in quasi metric spaces and related fixed point theorems, J. Inequal. Appl., 2014, 2014:36.

[10] S.A. Chen, W. Li, D. Zou, S.B. Chen, Fixed point theorems in quasi-metric spaces, Proc. of the Sixth International Conference on Machine Learning and Cybernetics, Hong Kong, 19-22 August 2007, 2499-2504.

[11] Şt. Cobzaş, Functional Analysis in Asymmetric Normed Spaces, Frontiers in Mathematics, Birkhäuser/Springer Basel, 2013. x+219 pp.

[12] N.V. Dung, Remarks on quasi-metric spaces, Miskolc Math. Notes, 15(2014), no. 2, 401-422.

[13] E. Karapınar, S. Czerwik, H. Aydi, $(\alpha, \psi)$-Meir-Keeler contraction mappings in generalized b-metric spaces, J. Funct. Spaces, 2018(2018), art. ID 3264620, 4 pages.

[14] M. Khan, M. Swaleh, S. Sessa, Fixed point theorems by altering distances between the points, Bull. Aust. Math. Soc., 30(1984), 1-9.

[15] N. Mlaiki, N. Taş, N.Y. Özgür, On the fixed-circle problem and Khan type contractions, Axioms, $\mathbf{7}(2018), 80$.

[16] A. Mukheimer, $\alpha-\psi-\phi$-contractive mappings in ordered partial b-metric spaces, J. Nonlinear Sci. Appl., 7(2014), no. 3, 168-179.

[17] N.Y. Özgür, Fixed-disc results via simulation functions, Turkish J. Math. 43(2019), no. 6, 2794-2805.

[18] N.Y. Özgür, N. Taş, Some fixed-circle theorems on metric spaces, Bull. Malays. Math. Sci. Soc., 42(2019), no. 4, 1433-1449.

[19] N.Y. Özgür, N. Taş, Some fixed-circle theorems and discontinuity at fixed circle, AIP Conference Proceedings, 1926, 020048 (2018).

[20] R.P. Pant, N.Y. Özgür, N. Taş, On discontinuity problem at fixed point, Bull. Malays. Math. Sci. Soc., 43(2020), no. 1, 499-517.

[21] V.Ć. Rajić, Some remarks on G-metric spaces and fixed point theorems, Int. J. Anal. Appl., 5(2014), no. 1, 102-114.

[22] I.L. Reilly, P.V. Subrahmanyam, M.K. Vamanamurthy, Cauchy sequences in quasi-pseudometric spaces, Monatsh. Math., 93(1982), 127-140.

[23] N. Shahzad, O. Valero, Fixed point theorems in quasi-metric spaces and the specialization partial order, Fixed Point Theory, 19(2018), no. 2, 733-749.

[24] M.B. Smyth, Completeness of quasi-uniform and syntopological spaces, J. London Math. Soc., 49(1994), 385-400.

[25] N. Taş, N.Y. Özgür, N. Mlaiki, New types of $F_{C}$-contractions and the fixed-circle problem, Mathematics, 6(2018), no. 10, 188.

[26] A. Tomar, R. Sharma, Some coincidence and common fixed point theorems concerning Fcontraction and applications, J. Int. Math. Virtual Inst., 8(2018), 181-198.

[27] D. Wardowski, Fixed points of a new type of contractive mappings in complete metric spaces, Fixed Point Theory Appl., 2012, 2012:94.

[28] W.A. Wilson, On quasi-metric spaces, Amer. J. Math., 53(1931), no. 3, 675-684.

Received: January 10, 2019; Accepted: May 8, 2019. 